\title{
Contralateral enhancement and suppression of vibrotactile sensation
}

\author{
GEORGE A. GESCHEIDER \\ Hamilton College, Clinton, New York \\ and \\ RONALD T. VERRILLO \\ Syracuse University, Syracuse, New York
}

\begin{abstract}
A peychophysical matching procedure was used to measure the effect of a conditioning stimulus on the vibrotactile sensation magnitude of a test stimulus. When both stimuli were applied to the thenar eminence of the same hand, the conditioning stimulus enhanced the sensation magnitude of the test stimulus. Enhancement was also observed when the test stimulus was on the thenar eminence and the conditioning stimulus was either on the contralateral thenar eminence, the ipsilateral middle finger, or the contralateral middle finger. When the conditioning and test stimuli were applied to separate sites, enhancement was maximal when $\Delta t$ between the stimuli was $150 \mathrm{msec}$. At $\Delta$ ts less than $100 \mathrm{msec}$, suppression was observed. Enhancement and suppression were observed only when the frequencies of the two stimuli were within the same vibrotactile information processing channel.
\end{abstract}

The sensation magnitude of a tactile stimulus may be enhanced or suppressed by preceding or following it with another tactile stimulus (Gescheider, Verrillo, Capraro, \& Hamer, 1977; Verrillo \& Gescheider, 1975, 1976, 1979a, 1979b). In these experiments, enhancement and suppression were measured by a psychophysical matching technique in which the subject was required to adjust the intensity of a comparison stimulus so that its subjective magnitude was equal to that of a test stimulus presented in the presence or in the absence of a conditioning stimulus. The differences between the match to the test stimulus alone and to the test stimulus in the presence of the conditioning stimulus were attributed to the effects of the conditioning stimulus and, thus, provided a measure of enhancement and suppression.

This method has been used in auditory experiments which investigated sensory interactions between successive stimuli (Bauer, Elmasian, \& Galambos, 1975; Elmasian, Galambos, \& Bernheim, 1980; Galambos, Bauer, Picton, Squires, \& Squires, 1972; Irwin \& Zwislocki, 1971; Zwislocki, Ketkar, Cannon, \& Nodar, 1974; Zwislocki \& Sokolich, 1974). In both touch and hearing, the interactive effects of double stimulation diminish as the time interval between successive stimuli increases. At $\Delta$ ts greater than $\mathbf{4 0 0}$ or $500 \mathrm{msec}$, no enhancement or suppression is observed. For both hearing and touch, enhancement

This research was supported by Grant NS-09940 from the National Institutes of Health, U.S. Department of Health and Human Services. G. A. Gescheider's mailing address is: Department of Psychology, Hamilton College, Clinton, New York 13323. is maximal when the frequencies of the two stimuli are identical. This finding was interpreted to indicate that presenting two tactile stimuli to different receptor populations may be analogous to presenting auditory stimuli in two widely separated critical bands, whereas presenting tactile stimuli to the same receptor population may be analogous to presenting auditory stimuli within the same critical band.

In our experiments on vibrotactile enhancement, we have consistently found that a conditioning stimulus of the same frequency, and applied to the same test site as a weaker test stimulus, will enhance the sensation magnitude of that test stimulus (Gescheider et al., 1977; Verrillo \& Gescheider, 1975). If, however, the test and conditioning stimuli are applied to different test sites, such as the thenar eminence and fingertip, sensation magnitude of the test stimulus may be suppressed when $\Delta t$ is very short $(0$ to $75 \mathrm{msec})$ and enhanced when it is longer (100 to $500 \mathrm{msec}$ ) (Verrillo \& Gescheider, 1976, 1979a). This result suggests that the psychophysically measured interaction effects between successive tactile stimuli are mediated by both inhibitory and excitatory neural processes. Since these neural processes are known to exist at virtually every level of the nervous system, we cannot be certain where in the nervous system enhancement and suppression take place.

In an attempt to gain information on the locus of suppression and enhancement, in the present study comparisons were made between results obtained when the conditioning stimulus was ipsilateral and when it was contralateral to the test stimulus. It was reasoned that, if enhancement and/or suppression 
occurs exclusively in the peripheral nervous system, the conditioning stimulus would have an effect on the sensation magnitude of the test stimulus only when the two stimuli were presented to the same test site or to ipsilateral test sites of close spatial proximity. If, however, enhancement and/or suppression occurs exclusively within the central nervous system or within both the central and peripheral nervous systems, some interactions might occur when the conditioning stimulus and test stimulus are presented to contralateral sides of the body.

\section{METHOD}

In all experiments, data were obtained from six right-handed subjects. Training consisted of several sessions in which the subjects made matching judgments for pairs of stimuli separated by various time intervals.

Sinusoidal vibratory stimuli were delivered to the thenar eminence of one or both hands, or sequentially to the thenar eminence and the distal pad of the middle finger, depending upon the experiment. A $2.9-\mathrm{cm}^{2}$ contactor was used for the thenar placement, and a $1.3-\mathrm{cm}$ contactor was used for the finger. The vibrator was adjusted at the start of each session so that the contactor pressed $.5 \mathrm{~mm}$ beyond contact with the surface of the skin. The vibratory stimulus was restricted to the region of the contactor by using a rigid surround upon which the subject's hand rested. Vibration amplitude was measured with a calibrated accelerometer mounted on the moving element of the vibrator, and all measurements were expressed in decibels re $1.0-\mu \mathrm{m}$ peak displacement of the contactor. All measurements were made while the subject's hand was in the test position. Stimuli were $20 \mathrm{msec}$ in duration measured at half-power points, had a $25-\mathrm{msec}$ rise/fall time, and had approximately Gaussian envelopes.

The subject and the vibrator assembly were located in a booth to provide isolation from vibrations of the building and sound in the laboratory. The sound of the vibrator was masked by a continuous, narrow-band noise centered at the frequency of the vibratory stimuli.

The procedure for measuring enhancement and suppression has been described and illustrated in previous publications (Gescheider et al., 1977; Verrillo \& Gescheider, 1975). The time interval between the onsets of the conditioning and test stimuli $(\Delta t)$ was varied over a range of 0 to $500 \mathrm{msec}$. The comparison stimulus was presented $750 \mathrm{msec}$ after the test stimulus. During a match, the stimulus sequence was repeated every $3.0 \mathrm{sec}$. The subjects were instructed to match the sensation magnitude of the comparison stimulus to the sensation magnitude of the test stimulus. The subjects used a bracketing procedure in which, on a single trial, they made several adjustments to the upper and lower limits of the match, after which they adjusted the intensity iteratively toward the center of this zone of uncertainty. Intensity of the comparison stimulus was controlled by two pedals which the subject pressed with the right and left feet. Each depression of the right pedal resulted in a 1.0-dB increase in intensity, whereas each depression of the left pedal produced a 1.0-dB decrease in intensity.

In our previous research on enhancement and suppression, subjects adjusted an unmarked knob attached to a continuously variable attenuator. In order to determine whether or not comparable results could be obtained with the two methods, an experiment was conducted in which six subjects made adjustments of the comparison stimulus using both procedures. The outcome of this experiment showed that virtually identical results are obtained with the two methods.

In every experiment, the subjects made three matches for each experimental condition. The order of presentation for stimulus pairs of various $\Delta$ ts was randomized within each experimental session, and the order of presentation of other variables was counterbalanced either over blocks of trials within a session or over sessions. Control matches, in which only the test and matching stimuli were presented, were made at the start of each session and again after the test matches were complete. Control matches were typically within $1.0 \mathrm{~dB}$ of each other. The effects of the presence of the conditioning stimulus upon the sensation magnitude of the test stimulus were expressed as the difference between experimental matches involving the pair (conditioning and test stimuli) and the average of the pre- and posttest control matches in which the conditioning stimulus was absent.

At the start of each session, the subject's threshold was measured by the method of limits and the intensity of the test stimulus was set at a sensation level of $10 \mathrm{~dB}$. The intensity of the conditioning stimulus was then adjusted until the subject reported that its sensation magnitude was the same as that of the test stimulus. Following this subjective match, the intensity of the conditioning stimulus was raised an additional $10 \mathrm{~dB}$.

\section{RESULTS}

In every experiment, each subject's results for each experimental condition were expressed as the average difference in decibels between the experimental and control matches. Since the results were very similar for all subjects, they were averaged. The graphs show the means of the data for the six subjects. The standard deviations were relatively small, ranging from .16 to $2.14 \mathrm{~dB}$, with a mean standard deviation of $.65 \mathrm{~dB}$. Standard deviations of $1.0 \mathrm{~dB}$ or less were obtained for $84 \%$ of the data points.

In the first experiment, stimuli were applied to the thenar eminence of one hand and a comparison was made of results obtained when the subject adjusted the intensity of the comparison stimulus by manipulating a knob with results obtained by the footpedal method. In this experiment, all three vibration bursts had a frequency of $300 \mathrm{~Hz}$. Virtually identical results were obtained with the two methods of adjusting stimulus intensity, and the same results were obtained for the right and left hands. In all conditions, the effect of the conditioning stimulus gradually decays to zero within about $500 \mathrm{msec}$. This finding agrees with the results of Gescheider et al. (1977) and Verrillo and Gescheider (1975), who reported that, when both the conditioning and test stimuli were presented to the thenar eminence, enhancement was maximal at the shortest $\Delta$ ts and diminished to zero as $\Delta t$ approached $500 \mathrm{msec}$. That enhancement may be a general sensory phenomenon with similar characteristics in other sensory modalities is suggested by the fact that loudness enhancement for $1,000-\mathrm{Hz}$ tone bursts has been found to decay at the same rate (Zwislocki \& Sokolich, 1974).

In the remaining experiments, the subject used the foot-pedal method to adjust stimulus intensity and the conditioning and test stimuli were on different sites. In Figure 1 are plotted the results of an experiment in which the test stimuli were always on the 


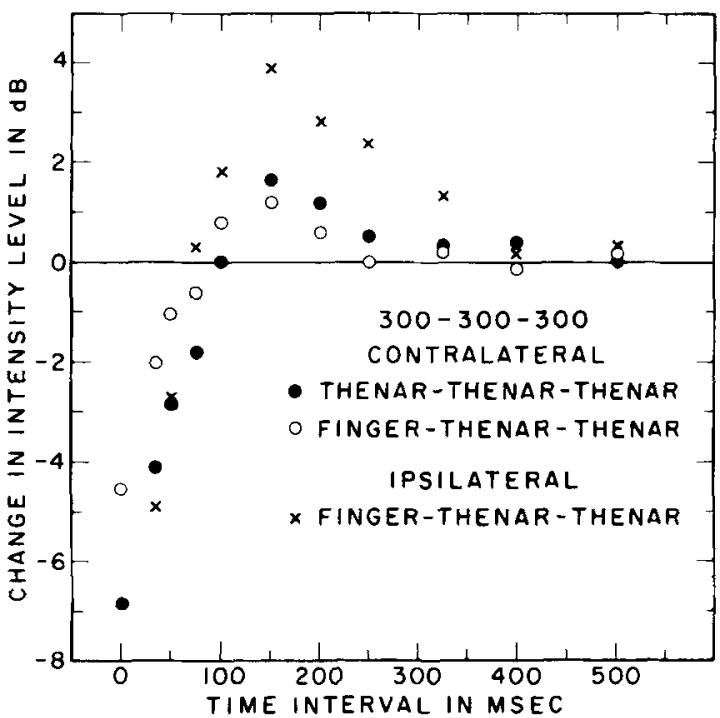

Figure 1. Change in the intensity level of the comparison stimulus when matched to the subjective intensity of the test stimulus as a function of the time interval between the conditioning and test stimuli. The test stimulus was delivered to the thenar eminence of the right hand, and the conditioning stimulus was delivered to the ipsilateral middle finger, the contralateral middle finger, or the contralateral thenar eminence. All three stimuli were presented to the thenar eminence in the thenar-thenar-thenar condition.

right thenar eminence and the conditioning stimulus was on the ipsilateral middle fingertip, the contralateral thenar eminence, or the contralateral middle fingertip. After the intensity of the conditioning stimulus was adjusted so that it had the same subjective magnitude as the 10-dB SL test stimulus, it was then raised $10 \mathrm{~dB}$. The frequency of all bursts was set at $300 \mathrm{~Hz}$. It can be seen in Figure 1 that, when the time interval separating the conditioning and test stimuli was less than approximately $75 \mathrm{msec}$, the presence of the prior stimulus caused a marked suppression in the subjective magnitude of the test stimulus. Suppression was followed by enhancement as $\Delta t$ was increased beyond $100 \mathrm{msec}$, with the greatest amount of enhancement occurring at $150 \mathrm{msec}$. The decay of enhancement effects between 150 and 500 msec resembles the decay observed when the conditioning and test stimuli are on the same site.

Although the greatest amounts of suppression and enhancement were observed when the conditioning stimulus was on the ipsilateral fingertip, it is important to note that a small, but reliable (observed in all subjects), amount of enhancement was observed when the test stimulus was either on the contralateral thenar eminence or contralateral fingertip. This finding indicates that enhancement is not purely a peripheral process but can originate within the central nervous system.

The effects of the conditioning stimulus were the same whether the test stimulus was on the left or the right hand (Figure 2). Thus, there is no evidence of hand dominance with enhancement or suppression as measured by this method.

An experiment was performed in which the test stimulus was on the thenar eminence of the right hand, the conditioning stimulus was on the contralateral thenar eminence, and stimulus frequency was either 25 or $300 \mathrm{~Hz}$. Essentially the same functions for suppression and enhancement were obtained for both low- and high-frequency stimulation. Verrillo and Gescheider (1975) have shown that enhancement effects were the same for both high- and low-frequency stimulation when both stimuli were applied to the thenar eminence and when one stimulus was applied to the thenar eminence and the other to the ipsilateral index fingertip. These and the present results support the hypothesis that enhancement and suppression effects occur in both the mechanoreceptive system innervated by Pacinian corpuscles (high frequency) and the system innervated by non-Pacinian mechanoreceptors (low frequency). Furthermore, the magnitudes and temporal properties of these effects appear to be identical for Pacinian and non-Pacinian stimulation.

The results of Verrillo and Gescheider $(1975,1976)$ also showed that, when the frequency of the conditioning stimulus is set to stimulate only one mechanoreceptive system and the frequency of the test stimulus is set to stimulate only the other system, the enhancement effect disappears. In the present study, an experiment was conducted to see if this result would also be obtained when the test stimulus was on

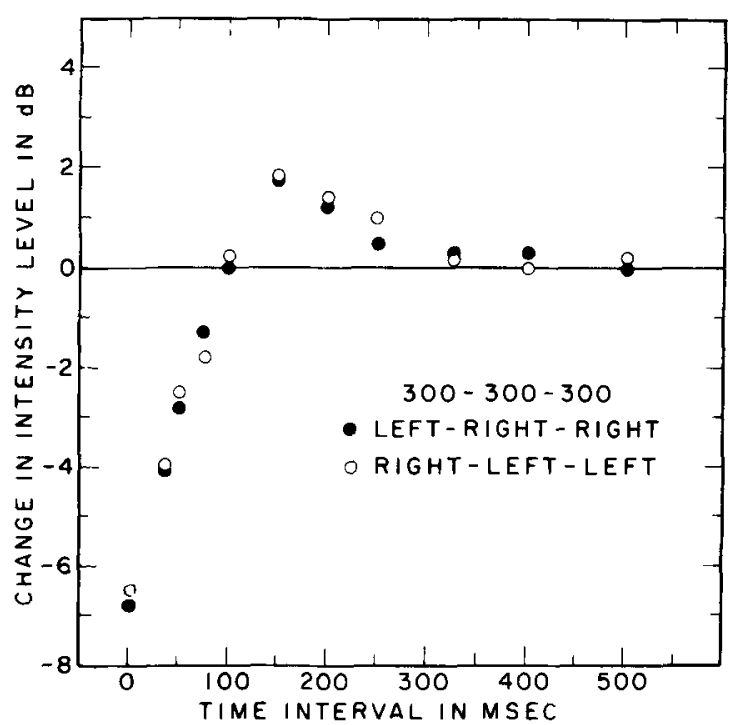

Figure 2. Change in the intensity level of the comparison stimulus when matched to the subjective intenalty of the test stimulas as a function of the time interval between the conditioning and test stimull. The teat stimulus was on the thenar eminence of elther the right or the left hand, and the conditioning stimulus was on the contralnternl thener eminence. 
the right thenar eminence and the conditioning stimulus was on the left thenar eminence. In this experiment, the frequencies of the conditioning and test stimuli were chosen such that one was within the sensitivity range of the non-Pacinian system $(25 \mathrm{~Hz})$ and the other was within the sensitivity range of the Pacinian system $(300 \mathrm{~Hz})$. The data in Figure 3 show that the contralateral enhancement and suppression effects do not occur when the conditioning and test stimuli are not set at frequencies within the sensitivity range of a single mechanoreceptive system.

In our final experiment, we examined the effects of a conditioning stimulus when it is present after, instead of before, the test stimulus. Backward enhancement and suppression of vibrotactile sensation was recently reported for cases in which the stimuli were applied to ipsilateral sites (Verrillo \& Gescheider, 1979a). In that study, backward suppression was measured when the test stimulus was on the thenar eminence of the right hand, and the conditioning stimulus was on the ipsilateral middle fingertip. Backward enhancement was observed when both stimuli were on the thenar eminence. In the backward enhancement procedure, the comparison stimulus was presented first. The test stimulus was presented $750 \mathrm{msec}$ after the comparison stimulus and was followed after a variable $\Delta t$ by the conditioning stimulus. The results plotted in Figure 4 show that when the conditioning and test stimuli were the same frequency, either 25 or $300 \mathrm{~Hz}$, the conditioning stimulus substantially suppressed the sensation magnitude of the test stimulus, but when the frequencies of the test and conditioning stimuli were 25 and $300 \mathrm{~Hz}$, respectively, neither enhancement nor suppression was observed. These data are nearly identical to those of experiments in which backward effects were measured for two-site stimulation on the same hand (Verrillo \& Gescheider, 1979a). In those experiments,

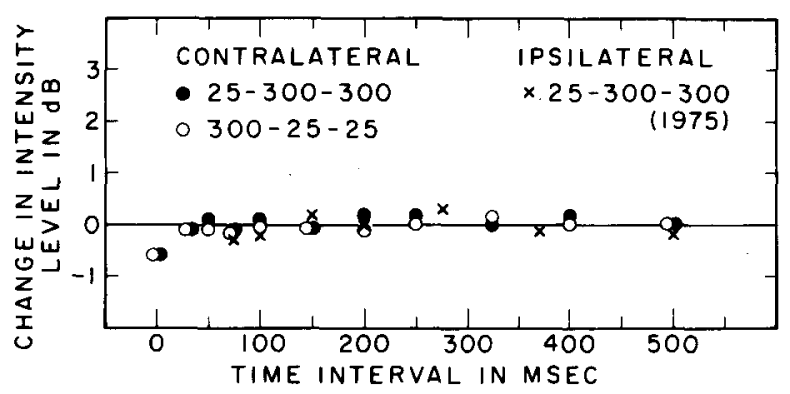

Figure 3. Change in the intensity level of the comparison stimulus when matched to the subjective intensity of the test stimulus as a function of the time interval between the conditioning and test stimull. Frequencies were chosen so that the conditioning stimulus exclusively stimulated one mechanoreceptive system, while the test stimulus excluaively stimulated the other system. The data from the Ipailateral 25-300-300-Hz condition $(x)$ are from Verrillo and Gescheider (1975).

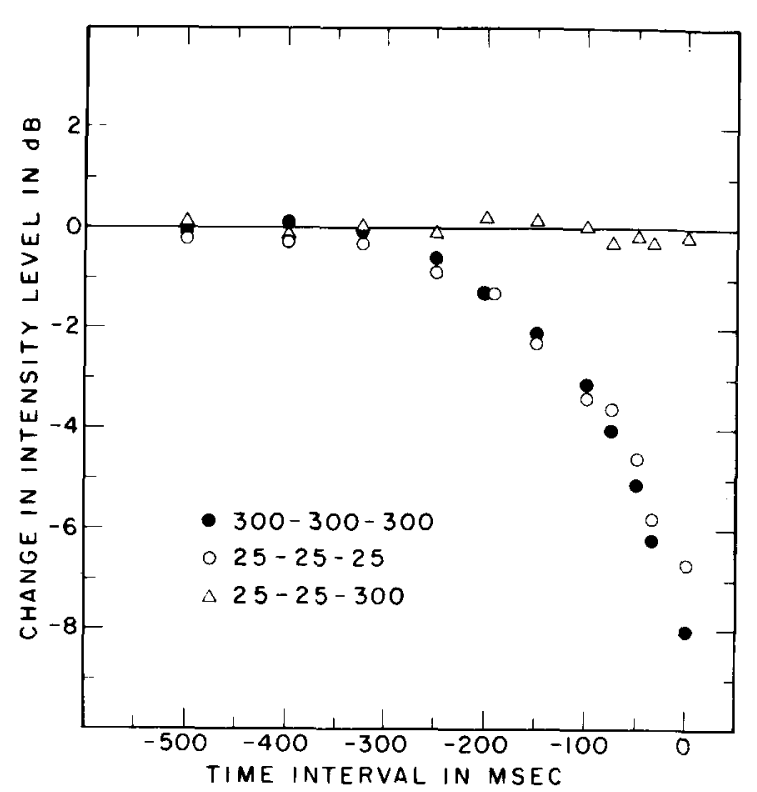

Figure 4. Change in the Intensity level of the comparison stimulus when matched to the subjective intensity of the test stimulus as a function of the time interval between the conditioning and test stimull. The conditioning stimulus was presented after the test stimulus. Frequencies were chosen so that the conditioning and test stimull stimulated the same (300-300-300 Hz, 25-25-25 Hz) or different (25-25-300 Hz) mechanoreceptive systems.

substantial suppression and no enhancement were found when the frequencies of test and conditioning stimuli were within the same vibrotactile channel. It was also found that neither enhancement nor suppression was found when the test and conditioning stimuli were isolated by frequency into different channels.

\section{DISCUSSION}

Several investigators have attempted to identify the locus and neural mechanism by which loudness enhancement is mediated in the auditory system. These attempts were based on the notion that traces of neural activity in the auditory nervous system may outlast the stimulus and, as a consequence of their interaction, cause loudness enhancement. Electrophysiological experiments were performed on cats (Bauer \& Galambos, 1975), and recordings were made in man with surface electrodes at the level of the brainstem (Bauer et al., 1975). In both studies, it was found that loudness enhancement could not be explained on the basis of the recorded electrophysiological responses. Neural activity of the test stimulus remained either unchanged or slightly depressed in response to prior stimulation by a conditioning stimulus. Thus, the exact locus of loudness enhancement remains unknown. It is reasonable to assume, however, that if loudness enhancement is due to the tem- 
poral integration of neural activity, it occurs in the central nervous system, since in peripheral auditory neurons neural firing stops with the termination of the stimulus (Kiang, 1965). Furthermore, it has been argued by Zwislocki (1960), in a paper on temporal auditory summation, that long-range integration must take place after the junction of the nerve tracts coming from the two ears, or otherwise the remarkable auditory sensitivity to dichotic time differences would be impossible.

In the case of vibrotaction, it is also unlikely that enhancement originates in the peripheral nervous system, since the time by which neural firing in peripheral nerves outlasts the stimulus (e.g., Knibestöl \& Vallbo, 1970) is much too short to account for enhancement effects which persist for 400 or $500 \mathrm{msec}$. Moreover, the fact that, in our psychophysical experiments, the rate of decay of enhancement with increasing $\Delta t$ between test and conditioning stimuli is approximately the same when the two stimuli are on the same site, on different ipsilateral sites, or on contralateral sites argues for some common central mediator of enhancement.

If enhancement is not due to persistence of peripheral neural activity, it may be due to temporal integration of persistent neural activity in afferent neurons in the central nervous system. This general notion was basic to Zwislocki's (1960) theory of auditory temporal summation. This theory accurately accounts for both auditory (Zwislocki, 1960) and cutaneous (Gescheider, 1976; Verrillo, 1965) data on how psychophysical detection thresholds decrease as the duration of the stimulus is increased. Although the time constants for temporal summation in detecting vibrotactile stimuli and vibrotactile enhancement are about the same $(200 \mathrm{msec})$, there are some problems with the temporal integration theory as an explanation of vibrotactile enhancement. One fact which is not consistent with a temporal integration explanation of enhancement is that enhancement occurs only when the conditioning stimulus is more intense than the test stimulus. This relationship is true for all levels of the conditioning stimulus (Gescheider et al., 1977). According to temporal integration theory, enhancement should be proportional to the intensity of the conditioning stimulus relative to absolute threshold, not relative to the varying intensity of the test stimulus. When the intensity of the conditioning stimulus is below that of the test stimulus, suppression, rather than enhancement, is observed (Verrillo \& Gescheider, 1975, 1976). This has also been reported for auditory enhancement experiments (e.g., Elmasian et al., 1980; Zwislocki \& Sokolich, 1974). Errors of judgment tend to be in the direction of the conditioning stimulus. If the conditioning stimulus is stronger than the test stimulus, the test stimulus will be judged stronger than when it is presented alone, and if the conditioning stimulus is weaker than the test stimulus, the test stimulus will be judged to be weaker than when presented alone.

Another problem with the temporal integration theory of enhancement is the fact that there is little, if any, temporal summation observed in experiments in which detection thresholds are measured for lowfrequency stimuli of variable duration (Gescheider, 1976; Gescheider et al., 1977). Nor is there any evidence for temporal summation above threshold when subjects judge the sensation magnitude of low-frequency vibration of variable duration (Gescheider, 1976). It appears that some mechanism other than temporal integration of neural activity is needed to explain why enhancement occurs with these low-frequency stimuli. Thus, at the present time there is no adequate theory of sensory enhancement.

Although we do not yet understand the exact neural mechanism for enhancement, the demonstration in the present study of contralateral enhancement does have implications for the neural basis of the perception of vibration. That there are at least two classes of mechanoreceptors which mediate the detection of vibration is well established (Mountcastle, LaMotte, \& Carli, 1972; Talbot, Darian-Smith, Kornhuber, \& Mountcastle, 1968; Verrillo, 1963, 1966a, 1966b, 1968). One class of mechanoreceptor responds best to low-frequency vibration, while the other (Pacinian corpuscles) responds best to higher frequencies of vibration. Through psychophysical experiments on selective adaptation (Gescheider, Frisina, \& Verrillo, 1979; Verrillo \& Gescheider, 1977), enhancement (Gescheider et al., 1977; Verrillo \& Gescheider, 1975, 1976), and masking (Labs, Gescheider, Fay, \& Lyons, 1978; Hamer \& Verrillo, Note 1), the two classes of mechanoreceptors have been identified as the input stages for two distinct information-processing channels. For example, vibrotactile adaptation, enhancement, and masking occur only when the conditioning and test stimuli are close enough in frequency to affect the same receptor system. That these two channels have specific representation in the central nervous system is indicated by the discovery of single cells in the somatosensory cortex that have response patterns characteristic of Pacinian corpuscles and cells with response patterns characteristic of nonPacinian mechanoreceptors (Ferrington \& Rowe, 1980; Mountcastle, Talbot, Sakata, \& Hyvärinen, 1969). The finding that contralateral enhancement occurs when the frequencies of the test and conditioning stimuli are within the same vibrotactile channel but not when they are in different channels suggests that the integrity of these channels remains intact as information is transmitted from one hemisphere of the cortex to the other. Neurophysiological support for this hypothesis comes from studies which 
indicate that there may be spatially separate areas on the somatosensory cortex for the representation of Pacinian and non-Pacinian inputs (Paul, Goodman, \& Merzenich, 1972) and different cytoarchitecture of Pacinian and non-Pacinian cells (Bennett, Ferrington, \& Rowe, 1980; Ferrington \& Rowe, 1980; Hyvarinen \& Poranen, 1978). Thus, both psychophysical and physiological evidence exists for the concept of at least two highly specific channels for the perception of vibration. These separate channels appear to remain intact at all levels of the nervous system.

\section{REFERENCE NOTE}

1. Hamer, R. D., \& Verrillo, R. T. Effect of sinusoidal maskers on vibrotactile information processing channels (Tech. Rep. ISR-20, Institute for Sensory Research). Syracuse, N.Y: Syracuse University, February 1975.

\section{REFERENCES}

Bauer, J. W., Elmasian, R., \& Galambos, R. Loudness enhancement in man: $I$. Brainstem evoked response correlates. Journal of the Acoustical Society of America, 1975, 57, 165-171.

Bauer, J. W., \& Galambos, R. Evoked potentials in cat auditory nerve: Suppression by prior tonal stimulation. Perception \& Psychophysics, 1975, 17, 43-47.

Bennet, R. E., Ferrington, D. G., \& Rowe, M. Tactile neuron classes within second somatosensory areas (SII) of cat cerebral cortex. Journal of Neurophysiology, 1980, 43, 292-309.

Elmasian, R., Galambos, R., \& Bernheim, A., Jr. Loudness enhancement and decrement in four paradigms. Journal of the Acoustical Society of America, 1980, 67, 601-607.

Ferrington, D. G., \& Rowe, M. Differential contributions to coding of cutaneous vibratory information by cortical somatosensory areas I and II. Journal of Neurophysiology, 1980,43, 310-331.

Galambos, R., Bauer, J., Picton, T., Squires, K., \& Squires, N. Loudness enhancement following contralateral stimulation. Journal of the Acoustical Society of America, $1972,52,1127-1130$.

GESCHEIDER, G. A. Evidence in support of the duplex theory of mechanoreception. Sensory Processes, 1976, 1, 68-76.

Gescheider, G. A., Frisina, R. D., \& Verrillo, R. T. Selective adaptation of vibrotactile thresholds. Sensory Processes, $1979,3,37-48$.

Gescheider, G. A., Verrillo, R. T., Capraro, A. J., \& HAMER, R. D. Enhancement of vibrotactile sensation magnitude and predictions from the duplex model of mechanoreception. Sensory Processes, 1977, 1, 187-203.

Hyvïrinen, J., \& Poranen, A. Receptive field integration and submodality convergence in the hand area of the post-central gyrus of the adult monkey. Journal of Physiology (London), 1978, 283, 539-556.

IRwiN, R. J., \& ZwislockI, J. J. Loudness effects in pairs of tone bursts. Perception \& Psychophysics, 1971, 10, 189-192.

KIANG, N. Y.-S. Discharge patterns of single fibers in the cat's auditory nerve (M.I.T. Monograph 35). Cambridge: M.I.T. Press, 1965.
KNIBEStöl, M., \& VRlLbo, A. B. Single unit analysis of mechanoreceptor activity from the human glabrous skin. Acta Physiologica Scandinavica, 1970, 80, 178-195.

Labs, S. M., Gescheiden, G. A., FAy, R. R., \& Lyons, C. H. Psychophysical tuning curves in vibrotaction. Sensory Processes, 1978, 2, 231-247.

Mountcastle, V. B., LaMotte, R. H., \& Carli, G. Detection thresholds for stimuli in humans and monkeys: Comparison with threshold events in mechanoreceptive afferent nerve fibers innervating the monkey hand. Journal of Neurophysiology, $1972,35,122-136$.

Mountcastle, V. B., Talbot, W. H., Sakata, H., \& HYvärinen, J. Cortical neuronal mechanisms in fluttervibration studied in unanesthetized monkeys. Neuronal periodicity and frequency discrimination. Journal of Neurophysiology, , 1969, 32, 452-484.

Paul, R. L., Goodman, H., \& Menzenich, M. Alterations in mechanoreceptor input to Brodmann's areas 1 and 3 of the postcentral hand area of Macaca mulata after nerve section and regeneration. Brain Research, 1972, 39, 1-19.

Talbot, W. H., Darian-Smith, I., Kornhuber, H. H., \& Mountcastue, V. B. The sense of flutter-vibration: Comparison of the human capacity with response patterns of mechanoreceptive afferents in the monkey hand. Journal of Neurophysiology, 1968, 31, 301-334.

Verrillo, R. T. Effect of contactor area on the vibrotactile threshold. Journal of the Acoustical Society of America, 1963, 35, 1962-1966.

VERRILLO, R. T. Temporal summation and vibrotactile sensitivity. Journal of the Acoustical Society of America, 1965, 37, 843-846.

Verrillo, R. T. Specificity of a cutaneous receptor. Perception \& Psychophysics, 1966, 1, 149-153. (a)

Veraillo, R. T. Vibrotactile sensitivity and the frequency response of the Pacinian corpuscle. Psychonomic Science, 1966, 4, 135-136. (b)

VerRitLo, R. T. A duplex mechanism of vibrotaction. In D. R. Kenshalo (Ed.), The skin senses. Springfield, Ill: Thomas, 1968.

Verrillo, R. T., \& Gescheider, G. A. Enhancement and summation in the perception of two successive vibrotactile stimuli. Perception \& Psychophysics, 1975, 18, 128-136.

Verrillo, R. T., \& Gescheider, G. A. Effect of double ipsilateral stimulation on vibrotactile sensation. Sensory Processes, 1976, 1, 127-137.

Verrillo, R. T., \& Gescheider, G. A. Effect of prior stimulation on vibrotactile thresholds. Sensory Processes, 1977, 1, 292-300.

Verrillo, R. T., \& Gescheider, G. A. Backward enhancement and suppression of vibrotactile sensation. Sensory Processes, 1979, 3, 249-260. (a)

Verrillo, R. T., \& Gescheider, G. A. Psychophysical measurements of enhancement, suppression, and surface-gradient effects in vibration. In D. R. Kenshalo (Ed.), Sensory functions of the skin of humans. New York: Plenum, 1979. (b)

Zwislock1, J. J. Theory of temporal auditory summation. Journal of the Acoustical Society of America, 1960, 32, 1046-1060.

Zwislocki, J. J., Ketkar, I., Cannon, M. W., \& Nodar, R. H. Loudness enhancement and summation in pairs of short sound bursts. Perception \& Psychophysics, 1974, 16, 91-100.

Zwislocki, J. J., \& Sokolich, W. G. On loudness enhancement of a tone burst by a preceding tone burst. Perception \& Psychophysics, 1974, 16, 87-90.

(Manuscript received March 8, 1982: accepted for publication March 11, 1982.) 